\title{
Rejoinder on: Single- and two-stage cross-sectional and time series benchmarking procedures for small area estimation
}

\author{
Danny Pfeffermann • Anna Sikov • Richard Tiller
}

Published online: 21 October 2014

(C) Sociedad de Estadística e Investigación Operativa 2014

We are very grateful to the discussants for their insightful comments and suggestions, which supplement the discussion in the paper and thereby enhance its merits. In what follows we respond to each discussion separately.

\section{Reply to Dr. Bell}

1. It is true of course that when an external benchmark that contains sampling error is modeled along with the series under consideration, maximum variance reduction is achieved by deriving the optimal estimators from the extended model, without forcing exact agreement with the benchmark. However, our experience shows that at least in official statistics, there is always the requirement of exact agreement between the small area estimators and the direct national or sub-national estimators, as implied by the requirement for consistency of publication. The consistency of publication also dictates the benchmarking equation used, for example, that the simple (unweighted) sum of the estimates of the small area totals equals the direct

This rejoinder refers to the comments available at doi:10.1007/s11749-014-0382-6; doi:10.1007/ s11749-014-0384-4; doi:10.1007/s11749-014-0386-2; doi:10.1007/s11749-014-0400-8.

D. Pfeffermann (凶)

University of Southampton, Southampton, UK

e-mail: msdanny@soton.ac.uk

D. Pfeffermann

Central Bureau of Statistics, Jerusalem, Israel

D. Pfeffermann · A. Sikov

Hebrew University of Jerusalem, Jerusalem, Israel

R. Tiller

Bureau of Labour Statistics, Washington, DC, USA 
design-based estimate of the subnational total, irrespective of the variances of the model-dependent small area estimators before benchmarking.

2. You mention the use of annual benchmarking in business surveys, in which the monthly figures are benchmarked to more reliable annual figures. In fact, this was the practice also at the BLS in the USA using internal benchmarking, whereby the monthly model-based Labour Force estimates had been benchmarked to the annual mean of the direct design-based estimators. The obvious drawback of this kind of benchmarking is that it is "retrospective", after the monthly estimates have already been published. In theory, benchmarking to reliable annual estimates is expected to produce better trend and seasonally adjusted estimates compared to the use of the unbenchmarked estimators, but this was not always the case at the BLS where in some of the States the annual benchmarks still had relatively large sampling errors, which introduced ripples into the trend estimates of many of the series. Notice in this respect that when the annual benchmark consists of the sum of the monthly cross-sectional design-based benchmarks, (or the annual external benchmark is the sum of the monthly cross-sectional external benchmarks), monthly benchmarking will automatically satisfy also annual benchmarking.

3. You state in the second to last paragraph of your discussion that "... imposing external time series benchmarking constraints on a sequence of cross-sectional small area predictors could correct for misspecification of a small area regression model if some areas were consistently under-predicted and others were consistently over-predicted." Are you referring here to monthly benchmarking? This would not be the case with the use of ratio (pro-rata) benchmarking where the unbenchmarked estimators at any given time are either all increased or all decreased as a result of the benchmarking. Is there an external benchmarking method that has this desired property of simultaneously correcting in the right direction under- and over-estimators resulting from the use of a misspecified small area regression model?

\section{Reply to Professor Morales}

1. Thank you for the elegant derivation of the ratio (pro-rata) benchmarked estimator as the solution of a divergence measure that contains the chi-square function. This property of ratio benchmarking is new to us and probably to many other readers of this article.

2. You go into great detail of how to apply monthly time series benchmarking under the Rao and Yu (1994) small area model. The computations are quite involved and need to be repeated every time that new observations become available. Notice, however, that the Rao-Yu model is a special case of the state-space time series model defined by Eqs. (8)-(9) in the paper, so that the benchmarked estimators and their variances can be obtained using the procedure described in Sects. 3.2-3.3. Following your notation, the Rao-Yu model for area $d$ is,

$$
\begin{aligned}
y_{d t} & =\boldsymbol{x}_{d t} \boldsymbol{\beta}+u_{1, d}+u_{2, d t}+e_{d t} ; u_{1, d} \sim N\left(0, \sigma_{1}^{2}\right), e_{d t} \sim N\left(0, \sigma_{d t}^{2}\right) \\
u_{2, d t} & =\rho u_{2, d, t-1}+\varepsilon_{d t} ; \operatorname{Var}\left(\varepsilon_{d t}\right)=\sigma_{\varepsilon}^{2} .
\end{aligned}
$$


To write it as a linear state-space model, define $z_{d t}^{\prime}=\left(\boldsymbol{x}_{d t}^{\prime}, 1,1\right) ; \boldsymbol{\alpha}_{d t}=$ $\left(\boldsymbol{\beta}^{\prime}, u_{1, d}, u_{2, d t}\right)^{\prime}$. A convenient state-space representation is,

$$
\begin{aligned}
& y_{d t}=z_{d t} \boldsymbol{\alpha}_{d t}+e_{d t} ; \boldsymbol{\alpha}_{d t}=T_{d} \boldsymbol{\alpha}_{d, t-1}+\boldsymbol{\eta}_{d t} ; T_{d}=\left[\begin{array}{ccc}
I_{p} & 0 & 0 \\
\mathbf{0}_{p}^{\prime} & 0 & 0 \\
\mathbf{0}_{p}^{\prime} & 0 & \rho
\end{array}\right], \quad \eta_{d t}^{\prime}=\left(\boldsymbol{\eta}_{\beta t}^{\prime}, u_{1, d}, \varepsilon_{d t}\right), \\
& \operatorname{Var}\left[\boldsymbol{\eta}_{d t}\right]=\left[\begin{array}{lll}
0_{p} & & \\
& \sigma_{1}^{2} & \\
& & \sigma_{\varepsilon}^{2}
\end{array}\right]=Q
\end{aligned}
$$

$\mathbf{0}_{p}^{\prime}$ is the null vector of order $p=\operatorname{dim}(\beta)$ and $O_{p}$ is the square null matrix of order $p$. For time $t=1$, the model for $u_{2, d t}$ is changed to $u_{2, d t}=0+u_{2, d t} ; \operatorname{Var}\left(u_{2, d t}\right)=$ $\sigma_{\varepsilon}^{2} /\left(1-\rho^{2}\right)=\sigma_{2}^{2}$.

Remark Under your approach you estimate the model hyper-parameters $\left(\beta, \sigma_{1}^{2}\right.$, $\left.\sigma_{2}^{2}, \rho\right)$ so that they satisfy the benchmarking constraints, which is referred to in the literature as self (automatic) benchmarking. See Section 2 of the paper. Under our approach, we first estimate the hyper-parameters by fitting the model without benchmarking and then apply the benchmarking procedure.

3. You consider a simple two-stage benchmarking procedure which consists of first benchmarking the subdomain (second level) estimates to the corresponding national direct estimate and then summing the subdomain estimates in any given domain to obtain the benchmarked domain estimates. You remark that this procedure might reduce the bias of the unbenchmarked model-based predictors at the subdomain level but not at the domain level. Notice that following this procedure requires joint modeling of the survey estimates in all the areas, which as illustrated in Section 6 of the paper is not always feasible in a real-time production system with strict deadlines. Under our approach we follow a reverse order, that is, we first benchmark the domain estimates to the national estimate and then benchmark the subdomain estimates in each domain to the corresponding benchmarked domain estimate obtained in the first stage. Benchmarking in this order potentially corrects the bias of the unbenchmarked estimators at both levels of the hierarchy. See the concluding remarks in Section 8 for conditions under which our time series benchmarking approach is expected to reduce the bias of the unbenchmarked estimators in all, or at least most of the areas.

\section{Reply to Professors Steorts and Ugarte}

1. You raise questions about the validity of the model used by the BLS for the production of the employment and unemployment estimates. We have not discussed this important issue in the present article but the model is obviously tested regularly and occasionally updated, accounting for outliers, level shifts, possible changes in the sampling error autocorrelations, etc. See Harvey (1989), for recommended statespace model testing procedures. Your analysis of data from the SAIPE program is well taken and illustrates the need for careful model testing, but it obviously does 
not invalidate the time series models used by the BLS. We mention in this respect that while the use of penalized splines may potentially improve the robustness of the models to nonlinearity, our experience with the BLS data shows that the past history of the series has much more prediction power than the covariate. We believe that this is true for many other times series. Our final remark in this regard is that in the simulation study of the present article the data have been generated under particular models with certain pre-imposed model misspecifications and we are focusing on the robustness of the cross-sectional and time series estimators to these model misspecifications, rather than on model testing.

2. You mention Ghosh and Steorts (2013) as an alternative method of two-stage time series benchmarking but as far as we can tell, this article only considers crosssectional benchmarking. As discussed and illustrated in Section 6, computing the benchmarked estimators at both levels in a single run is not feasible in a monthly production system under the times series models used by the BLS. In any case, the relevant comparison between the methods in the present context is with respect to the benchmarking procedures applied and not with respect to the models fitted to the data. The choice between the Frequentist and the Bayesian approaches is always an intriguing issue, but as you also mention, as far as computational issues are concerned, it is not clear which approach is less computationally intensive.

3. You argue that "instead of making the domain estimate a hard external constraint, it should be incorporated into a probabilistic model along with the subdomain data", adding that "it is hard to believe that the direct domain estimates used in the present article are fixed and without error" and that the "uncertainty in these external quantities should be incorporated into the benchmarking". But this is exactly what we are doing. See Eqs. (14) and (31) in the paper. We do impose exact benchmarking such that the benchmarked estimators add to the benchmark, which as we mentioned in our reply to Dr. Bell is required in the production of official statistics to satisfy consistency of publication. However, we account for the error of the benchmark when computing the variances of the benchmarked estimators for any given time point, and also when computing the benchmarked estimators in subsequent time points.

4. You mention the problem of small sample sizes and out of sample areas. Small sample sizes make the use of time series models that borrow information from the past even more crucial. Our procedure can be extended to obtain estimates for areas with no sample at isolated time points. Estimation of the means of areas with no samples at all the time points requires modeling the cross-sectional relationships between the true area means or their components (trend, seasonal effects), which may also improve the estimation in areas with samples.

\section{Reply to Dr. Ha and Professor Lahiri}

1. You mention that the additive benchmarked estimator given by Eq. (3) of the paper is expected to be design-consistent for a self-weighting design under certain regularity conditions. This statement requires some further examination. In our paper we stated that if the correction coefficient $\lambda_{d}$ in (3) tends to zero as the 
area sample size increases, which would be the case for commonly used choices of the coefficients $\left\{\varphi_{d}\right\}$, the benchmarked estimator will converge in probability to the model-dependent BLUP, which in turn is consistent for the true area mean under the model. For the Fay-Herriot model under which the additive benchmarked estimator in (3) has been derived, this implies also design consistency even if the model is misspecified (e.g., an important covariate is missing from the model or not transformed properly), because as the area sample size increases, the (shrinkage) weight attached to the direct estimator in the expression of the BLUP under the (misspecified) model tends to one and the direct estimator is generally designconsistent. However, under models for which the BLUP or the best estimator does not converge to the direct estimator as the area sample size increases, the designconsistency property may no longer hold even though $\lambda_{d}$ still tends to zero as the area sample size increases.

2. Further to the previous comment, you emphasize in your discussion designconsistency under self-weighting designs. If by self-weighting you mean equal ultimate (unit) sample selection probabilities, then we do not see why this is required. As the discussion in the previous comment implies, the design-consistency does not require equal selection probabilities and only requires that the direct estimator is design-consistent for the true area mean.

3. Thank you for the derivation of the raked benchmarked estimator, which is an interesting addition to the growing family of benchmarked estimators.

4. You mention the use of self-benchmarked estimators, referencing the work of You and Rao (2002). In the paper we review also the work of Wang et al. (2008) where a different self-benchmarked estimator is proposed. Professor Morales proposes a self-benchmarked estimator under the Rao and Yu (1994) model in his discussion of our paper.

5. You seem to propose the use of cross-sectional benchmarking in conjunction with the use of time series models, but if we interpret your comment correctly, this is exactly what our single-stage and two-stage time series benchmarking procedures are all about. See Eqs. (13) and (30) in the paper which are cross-sectional constraints. Our procedure produces also the variances of the benchmarked estimators under the model for given model parameters. The use of parametric bootstrap as proposed in Pfeffermann and Tiller (Journal of Time series analysis, 26, pp. 893916) produces variance estimators of correct order when using estimated model parameters.

6. Thank you for mentioning the pioneering work of Louis (1984) on quadratic benchmarking under the Bayesian and empirical Bayes methodology. We should have included this work in our review. 